controversy (such as, phenylketonuria, Down's syndrome, Tay-Sachs), and most would agree that relatively mild homozygotic conditions can depress the IQ of afflicted persons (children who suffer from sickle cell anaemia or cystic fibrosis may test lower for IQ, on average, simply because they miss a great deal of school). Does this mean that such children have genetically heritable IQ? Does it mean, in the formal sense, that the disparity in IQ between them and normal children is attributable to variance in genes? Of course, it means exactly that - and yet the genes for beta haemoglobin and regulation of mucous production are not "IQ genes".

I am certain that my results on an IQ test would suffer if I were tested while I had a head cold. Imagine two boys reared in the same environment who are genetically identical except that one has an inherited sensitivity to ragweed. If they lived in a high ragweed area, such that the allergic one suffered from chronic congestion, would it be surprising to find that he tests slightly lower for IQ? Intelligence is a summary performance indicator, as distinct from an isolated component indicator (like how well a person's brain works), in the sense that suboptimal performance in any subsystem can have a negative impact. Variability in intelligence is perhaps analogous to the variability in horse power of a group of nominally identical engines, where quality of fuel represents the environmental component and fine tuning represents the genetic component. Performance is maximized when an engine is tuned for its operating conditions. A set of engines tuned in the tropics will not perform as well as when transported to Toronto. The question becomes, to what degree does the distribution of nonoptimal non-IQ-genes account for heritable IQ?

The relative optimality of a gene is often environmentally specified. Teasdale and Owen, like others before them, found that the heritable component of IQ could be determined by segregation of a few alleles. ("The results for intelligence conform closely to what would be predicted by a simple polygenic model of genetic transmission . . ."). There are two ways this could happen: (1) there may be a universal handful of IQ genes that segregate appropriately, or (2) in any given environment, there may be a handful of genes for each of any one of hundreds of traits that can have a negative impact on $\mathrm{IQ}$, and some of those handfuls segregate appropriately. If the former is the case, one wonders why alleles for superior IQ are not closer to fixation. Is there polymorphism for IQ because there were evolutionary environments in which intelligence was selected against?

Think about breathing through your nose in a steam bath. This is a genuine problem. Indeed, if you have a relatively long, thin, hairy nose (as typifies people from temperate zones and/or high altitudes - like Swedes and Ethiopians) it can be virtually impossible to pull enough air through that nose. But observe the steam bath breathing behaviour of someone with a wide, short, hairless nose (as typifies blacks and orientals indigenous to tropical wetlands). These people can breath superhumid air through their nose with no problem. Under the extreme condition of a steam bath, nose shape so strongly affects breathing efficiency that you can feel the difference by mechanically flaring your nostrils. Humans take over 10 million breaths a year. I submit that if we were all reared in steam baths, people with wide, short, hairless noses would have slightly higher mean IQ. This scenario is not silly because real noses in the real world are very differently adapted to conditions of humidity or dust. Among American racial and ethnic groups, only blacks and nonJapanese orientals have a conspicuous prevalence of lung disease ${ }^{3}$. Dust and airborne microorganisms can be a serious health hazard ${ }^{4}$. Noses have evolved accordingly. ${ }^{5-7}$

Can differences in nose shape (inherited) account for differences between black and white American IQ? It would be ridiculous to think so, but it should be considered equally ridiculous to assume that such factors have no effect. What about skin colour? More sunlight is required by dark skin to synthesize the same amount of vitamin D. Just as dark skin prevents hypervitaminosis-D in the tropics, so light skin facilitates D synthesis in temperate zones $^{8-10}$. The question becomes, how

\section{Raising the tone of DNA sequences}

SIR - We assessed the potential for gene jingles following a lunchtime conversation two years ago. Hayashi and Munkata (Nature 310, 96; 1984) appear to have used a purely arbitrary pitch-to-base assignment and ignored the complementary DNA strand altogether. We chose the notes D, A, F sharp, D, but have given some thought to base assignment of which there are 24 possibilities. We felt the complementary base triplet of each codon should produce a 'complementary' tune. Rather naively, we suspect, in musical terms, we took as complementary the quasi-mirror image of the notes on the stave (on the left of the figure). Eight of the possible 24 pitch-tobase assignments meet this requirement, but the assignment used by Hayashi and Munkata does not (on the right). many such factors are there? Dozens? Hundreds? More than enough to account for the portion of IQ variance which is heritable? Quite possibly. But this is not why the question is important.

The question is important because there are many factors that something could be done about if we knew what they were. People with dark skin in dark climates can get some extra sunshine. People with nondust-trapping noses can have humidifiers in their homes during winter and take care to wear a dust mask when doing a dusty job. Unfortunately, looking for genetic differences that incidentally affect IQ is politically unacceptable because such investigations tacitly acknowledge the existence of such differences.

It may seem naive to think that things like clean air and sunshine could ameliorate genetic differences that contribute to differences in IQ. Fortunately, naivete is also in the eye of the beholder.

Department of Anesthesiology,

JOHN HARTUNG

Downstate Medical Center.

State University of New York,

New York 11203, USA

1. Teasdale, T.W. \& Owen, D.R. Nature 309, 620-622 (1984)

2. Hartung, J. Curr. Anthropol 21, 131-132 (1980).

4. Cohen, D. Am J. Phys. Anthrop. 22, 375-382. (1979).

. Newman, M.T. Am. Anthropol. 55, 311-327 (1953)

6. Biasutti, R. Razze e Popoli della Terra (Unione Tipografico-Edi Traice, Torino, 1959).

7. Wolpoff, M.H. Am. J. Phys. Anthrool. 29, 405-423 (1968).

8. Loomis, W.F. Science 157, 501 -506 (1967).

9. Haddad, J.G. \& Hahn T.J. Nature 244, 515-516 (1973).

10. Robertson, I., Glekin, B.M., McIntosh, W.B., Lakhani, A \& Dunnigan, M.G. Proc. Nutr. Soc. 41, 243-250 (1982).

One of us (M.P.) sent short recorded DNA tunes to the other for the final selection of the pitch-to-base assignment by ear. No one assignment stood out as best, but the tunes of the promoter region CCAAT box were more pleasing musically than the 9-base repeat of collagen.

We are working towards the first public performance of 'Variations on a Cryptic Splice Junction' and the correspondence has given us renewed enthusiasm.

MARCUS PEMBREY Mothercare Unit of Paediatric Genetics, Institute of Child Health, 30 Guilford Street, London WCI IGH
8 Chester Street,

London SWI, UK

DAVID HART

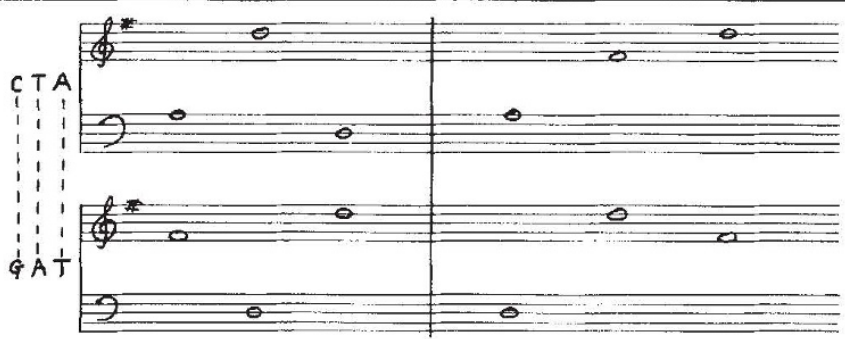

Base pairing by Pembrey and Hart (left) and Hayashi and Munkata (right). 